\title{
Management und Medizin post-Corona
}

\author{
Christof Schmitz ${ }^{a}$, Marcel Zwahlen ${ }^{b}$, Peter Berchtold ${ }^{a}$, \\ ${ }^{a}$ college M, Bern; ${ }^{b}$ ISPM, Universität Bern
}

Die Medizin hat in den letzten Monaten rasant über SARS-CoV-2 gelernt, die Bevölkerung über Epidemiologie, Statistik und die Vulnerabilität des Gesundheitssystems. Was hat das Management gelernt? Erfahrungen der Krisenzeit zeigen, was möglich ist, wenn Unsicherheit akzeptiert, interprofessionell zusammengearbeitet, fortlaufend miteinander adaptiert wird und Interessen hintangestellt werden, also «agil» gearbeitet wird. Daraus liesse sich lernen. Wir bleiben vorsichtig pessimistisch, was dieses Lernen betrifft.

Die COVID-Wochen hatten es für Spitäler in sich. Krisenstäbe, Sonderschichten, rasantes Lernen und intensives Arbeiten bei den einen fand sich ebenso wie nahezu Stillstand bei den anderen, die nichts mit COVID-19-Patienten zu tun hatten. Wir wollen hier den Blick auf die positiven Erfahrungen der Zusammenarbeit in dieser Zeit richten, die sich in mehreren Auswertungsrunden in der Schweiz und in Deutschland zeigten (unbenommen auch negativer Erfahrungen, die an verschiedenen Orten gemacht wurden). Dabei treten drei Aspekte besonders hervor: die Kooperationsbereitschaft unter Krisenbedingungen, die Möglichkeit (unerwartet) rascher Veränderungen und die Chance agilen Handelns.

- Kooperationsbereitschaft: Vielfach wurden aus den Spitälern sehr befriedigende Erfahrungen der Zusammenarbeit und ihrer Handlungsergebnisse berichtet. Die Krisenstäbe und ihre Subeinheiten agierten mehrheitlich, oftmals nach anfänglichem «Storming», produktiv, trafen sich fortlaufend und stimmten sich Schritt für Schritt ab. Man konnte sich einer hohen Bereitschaft zur (interprofessionellen) Zusammenarbeit erfreuen (es sei denn, die Verhältnisse von vorher waren so schlecht, dass es auch in der Krise nicht gelang, Brücken zu bauen). So konnten die IPS-Kapazitäten erfolgreich ausgebaut und konstruktiv auf die sich fortlaufende Veränderung der Lage reagiert werden. Einige involvierte Personen bedauerten teilweise gar die Auflösung der Krisenstäbe, weil sie selten solch gute Zusammenarbeit erlebt hätten.
- Rasche Veränderungen: Berichtet wurde ferner, wie viele Veränderungen rasch, ja, unerwartet rasch möglich wurden. Was sonst oft monatelanger Verhandlungen bedurfte, konnte verschiedentlich in einem Nachmittag entschieden werden. Viele Veränderungen gingen in erstaunlichem Tempo und unerwartet «smooth» vonstatten. Veränderungsbereitschaft war in hohem Masse gegeben, man kam zügig zu Entscheidungen und setzte unmittelbar um. Dabei erhielt man - oder nahm sich - (sonst ungewohnten) Freiraum, ohne der Verdächtigung ausgesetzt zu sein, lediglich Eigeninteressen wahrnehmen zu wollen.

\section{Wir wollen hier den Blick auf die positiven} Erfahrungen der Zusammenarbeit in dieser Zeit richten.

- Agiles Handeln: Die Krisenverarbeitung erfolgte weniger nach üblichen (Projekt-)Managementmethoden. Vielmehr ging es darum, den Erfordernissen der Situation zu gehorchen und rasche Anpassung und ein fortwährendes Lernen aus der Situation zu begünstigen. Die im Management zum Hype-Begriff gewordene «Agilität» meint im (nützlichen) Kern ein Arbeiten an komplexen Themen, das hochgradig iterativ, in fortlaufender, zielorientierter Abstimmung und typischerweise unter weitreichender Selbstorganisation cross-funktionaler (im Spital: interprofessioneller) Teams fungiert. Die vergangenen Wochen haben gezeigt, dass viele Spi- 
täler die Krise mit tendenziell agilen Prinzipien bewältigten, damit gute Ergebnisse erzielten und dies als sehr positiv erlebt wurde. In jedem Fall wurden bessere Ergebnisse erreicht, als wenn die üblichen Projektmanagement-Formen zur Anwendung gekommen wären.

\section{Normalfall und Krise}

Auffallend an dieser Beschreibung krisenbedingter Zusammenarbeit sind die eklatanten Unterschiede zum «Normalfall» des üblichen Funktionierens von Spitälern. Im Normalfall ist spitalübergreifende, veränderungsorientierte Zusammenarbeit vielfach holprig und gezeichnet von Eigeninteressen und Machtspielen wie von langen Bearbeitungs- und Entscheidungszyklen. Vieles davon gründet in den weiten Verästelungen der Spitalsysteme mit ihren vielen Fächern, Berufsgruppen, Perspektiven und sonstigen Fragmentierungen. Gleichwohl stellen sich mit Blick auf die COVID-Erfahrungen die Fragen, was den Unterschied ausmacht und was das beschriebene «agile» Arbeiten begünstigen könnte?

Wichtig sind, wenn wir den bisherigen Analysen folgen, vier Dimensionen: 1) Umgang mit (eingestandenem) Unwissen und Ungewissheit, 2) Orientieren an einem klaren "purpose», 3) Einbinden von (Einzel-) Interessen und 4) Einklammern der Ökonomie. Diese vier Dimensionen erlaubten es in der Krise, jenen "common ground" zu finden, der rasches und effektives kollektives Handeln erst erlaubt.

\section{Im Normalfall ist spitalübergreifende, verände- rungsorientierte Zusammenarbeit vielfach holprig.}

- Unwissen und Ungewissheit: Weder existierte Erfahrung mit dem SARS-CoV2-Virus, noch wusste irgendjemand exakt, wie die Behandlung dieser Krankheit und ein solch unerhörtes Hochfahren insbesondere der intensivmedizinischen Kapazitäten gelingen sollte. Auch wenn virologisches, intensivmedizinisches oder KatastrophenmanagementWissen vorhanden war, waren gleichzeitig Unwissen und Ungewissheit nicht zu leugnen. Das ist der Punkt: Nichts stärkt Zusammenarbeit mehr, als das Bewusstsein, dass alle ähnlich wenig wissen und dass man aufeinander angewiesen ist. Im Umkehrschluss heisst das: Nichts ist hinderlicher für Zusammenarbeit, als wenn wichtige Akteure meinen zu wissen, worum und wie es gehen soll. Dann setzen jene $\mathrm{K}(\mathrm{r})$ ämpfe ein, die so bekannt - und so ermüdend - sind und die jene endlosen Friktionen erzeugen, an denen im Normalfall so viele leiden.

\section{CAS Managing Medicine in Health Care \\ Organisations}

Medizinische Versorgung wird geplant, gestaltet, erbracht und bewirtschaftet. Dabei gilt es zahlreiche äussere wie innere Spannungen zu balancieren. Exakt das bedeutet Managing Medicine. Ein gutes Verständnis der Möglichkeiten wie der Grenzen des Managements und seiner Instrumente zu haben, ist heute für medizinisches Führungspersonal absolut wesentlich. Dieses CAS vermittelt zentrale Konzepte und Instrumente des Managements und der Steuerung medizinischer Organisationen.

Ein Angebot der medizinischen Fakultät der Universität Bern unter Leitung des Instituts für Sozial- und Präventivmedizin ISPM, durchgeführt in Zusammenarbeit mit college M

Das CAS richtet sich an Ärzte und Ärztinnen mit leitenden Funktionen sowie Führungskräfte anderer Berufsgruppen in Spitälern und weiteren Einrichtungen.

Detaillierte Informationen unter www.cas-managingmedicine.ch

- «Purpose»: Die (drohende) Krise vereinte unter einer gemeinsamen Aufgabe den Sinn oder, im aktuellen management-talk ausgedrückt, den «Purpose» sowohl der Organisation als auch der Professionen: die Gesundheitsversorgung der Bevölkerung. Es ging um ein medizinisch relevantes Problem, das von allen Beteiligten übereinstimmend geteilt wurde. Diese Kombination von Krise und «Purpose» half an vielen Orten jene produktive Kollektivität hervorzubringen, die es braucht, um mit dem Druck einer solchen Situation umgehen zu können.

- Der zeitliche, sachliche wie soziale Druck der Krise liess mindestens für eine bestimmte Zeit von den üblichen Interessen absehen. Die einzelnen Akteure mussten nicht so sehr die Interessen ihrer jeweiligen Gruppen oder Organisationseinheiten (oder auch ihre eigenen) repräsentieren, sondern konnten sich einer gemeinsamen Aufgabe widmen. Mit der Krise war ein Rahmen gespannt, in dem es weniger galt, eigenen Bestand zu sichern als eine gemeinsame Zukunft - die Bewältigung der Krise zu gewinnen.

- Für einige Wochen fiel zudem eine wichtige Perspektive weg, die den Alltag der Spitäler sonst so stark mit prägt, nämlich die der Ökonomie. Während ökonomisch-betriebswirtschaftliche Fragen sonst ständig mitspielen müssen (Was kosten dieses? Wieviel Ertrag bringt jenes?), stand für den Moment die medizinische Krisenbewältigung - der Purpose - völlig im Vordergrund. So wie die Gesamtgesellschaft in einer welthistorisch einzigartigen Weise einen Stillstand der Wirtschaft zu verhindern suchte, wurde auch in den Spitälern die Ökonomie ausser Kraft gesetzt - temporär. 


\section{Die Dramatik der Perspektivendifferenz}

Das Aufstrahlen des «Purpose» sowie das (temporäre) Wegklappen der ökonomischen Perspektive ermöglichte die Entschärfung einer Dramatik, die sonst besteht. Diese Dramatik ist der medizinischen Leistungserbringung inhärent und resultiert aus der Anforderung fortlaufend zwischen diagnostischen, therapeutischen, pflegerischen, managerialen, ökonomischen und politischen Perspektiven zu vermitteln und darüber hinaus diese in Abstimmung zu bringen. Diese Perspektivendifferenzen sind dramatischer Natur, weil sie grundsätzlich nicht ausgleichbar sind (Medizin $\neq$ Ökonomie $\neq$ Politik $\neq$ Pflege usw.) Sie sind dramatisch unterschiedlich. Auch wenn ihre Vermittlung ganz alltäglich und bei jeder Patientenbehandlung stattfindet, verbleibt vielfach ein Ringen um Relevanz oder sogar um Dominanz. Dieses Ringen war in den letzten Jahren insbesondere von der Auseinandersetzung zwischen der ökonomischen und der medizinischen Perspektive geprägt (Stichwort: Ökonomisierung der Medizin). Und exakt dieses dramatische Ringen war durch die Krise stillgelegt. Es ging um Medizinisches, Punkt.

\section{Lesson learned}

Diese Lektion lässt sich lernen: Agiles Handeln, also eines, das komplexe Aufgaben mit klar gesetzten, iterativen Schritten, gut gerahmten Abstimmungen und der Selbstorganisationsfähigkeiten cross-funktionaler (interprofessioneller) Teams effektiv bewältigt, kann dann umgesetzt werden, wenn es gelingt, den Fallstricken des Normalfalls zu entkommen. Agiles Handeln führt dann 1) zu guten Ergebnissen und 2) zu Zufriedenheit bei den Beteiligten. Im Management Talk blei- bend heisst das, dass Performance und Wellbeing erreicht werden. Und genau das ist es, worum es geht, wenn wir unser Wissen über Expertenorganisationen, Motivation und Leistung ernst nehmen wollten.

Für agiles Arbeiten in diesem Sinne braucht es - halten wir es nochmals fest - das gemeinsame Empfinden einer Dringlichkeit (zeitliche, sachliche oder soziale Relevanz) und die Aktualisierung des gemeinsam geteilten «Purpose». Das ist vielleicht die wichtigste Botschaft, die es wahrzunehmen gilt: Vor allem dann, wenn gemeinsam als medizinisch relevant Empfundenes im Vordergrund steht, werden solche produktiven Management-Formen funktionieren. Die Steigerung des EBITDA kann nicht primärer Purpose von Spitälern sein. Das ist keinerlei Aussage darüber, dass diese Kennzahl nicht wichtig ist, wenn die systemischen Rahmenbedingungen entsprechend gesetzt sind. Aber medizinisch relevant und den Sinn der Spital-Organisation und ihre Energien aktivierend sind Betriebswirtschaft und Ökonomie nur in indirekter Weise. Möglicherweise bietet die Coronoa-Krise eine Chance, den Purpose des Spitals und die damit verbundenen motivationalen Kräfte wieder einmal klarer zu kriegen. Für Betriebswirte mag das auf den ersten Blick etwas desillusionierend klingen, wiewohl es ihre Bedeutung nicht schmälert, sondern zeigt, wie anspruchsvoll ihre Beiträge in diesem Kontext sind. Für die Medizinerinnen und Mediziner aber erhebt sich die Frage, wo die mobilisierenden institutionsübergreifenden Zukunftsprojekte liegen? Da lassen sich Ideen entwickeln. Aber angesichts des spezialisierten Charakters der Medizin und der hohen Anforderungen an den Umgang mit der beschriebenen "Dramatik», ist vermutlich nicht zu viel Optimismus, eher ein "vorsichtiger Pessimismus» angebracht. Oder sollten wir uns täuschen?

\section{Das Wichtigste in Kürze}

- Die COVID-19-Krise hat aus Sicht der Autoren auch positive Erfahrungen mit sich gebracht, vor allem in Bezug auf die Kooperationsbereitschaft unter Krisenbedingungen, die Möglichkeit (unerwartet) rascher Veränderungen und die Chance agilen Handelns.

- Vier Dimensionen erlaubten in der Krise, einen «common ground" zu finden, der rasches und effektives kollektives Handeln erst erlaubt: Umgang mit (eingestandenem) Unwissen und Ungewissheit, Orientieren an einem klaren "purpose», Einbinden von (Einzel-)Interessen und Einklammern der Ökonomie.

- Agiles Handeln kann dann umgesetzt werden, wenn es gelingt, den Fallstricken des Normalfalls zu entkommen; dazu braucht es das gemeinsame Empfinden einer Dringlichkeit (zeitliche, sachliche oder soziale Relevanz) und die Aktualisierung des gemeinsam geteilten "purpose».

\section{L'essentiel en bref}

- De l'avis des auteurs, la crise du COVID-19 a également donné lieu à des expériences positives, notamment en matière d'ouverture à la coopération en conditions de crise, de possibilité de changements d'une rapidité inattendue et d'opportunités d'actions agiles.

- Quatre dimensions ont permis de trouver pendant la crise un "terrain d'entente», dont dépend toute action collective rapide et efficace: la gestion de l'ignorance et de l'incertitude (avouées), l'orientation vers un objectif clair, la prise en compte des intérêts (individuels) et la mise entre parenthèses de l'économie.

- L'action agile peut être mise en œuvre lorsque I'on parvient à déjouer les pièges du cas normal. Cela exige un sentiment partagé d'urgence (importance temporelle, matérielle ou sociale) et I'actualisation de l'objectif commun. 